\title{
The role of physiological elements in future therapies of rheumatoid arthritis. III. The role of the electromagnetic field in regulation of redox potential and life cycle of inflammatory cells*
}

\author{
Michał Gajewski ${ }^{1}$, Przemysław Rzodkiewicz ${ }^{1}$, Sławomir Maśliński ${ }^{2}$, Elżbieta Wojtecka-Łukasik ${ }^{1}$ \\ ${ }^{1}$ Department of Biochemistry and Molecular Biology, Institute of Rheumatology, Warsaw, Poland \\ ${ }^{2}$ Department of General and Experimental Pathology, Medical University of Warsaw, Poland
}

\begin{abstract}
Each material consisting of charged particles can be influenced by a magnetic field. Polarized particles play an essential role in almost all physiological processes. Locally generated electromagnetic fields several physiological processes within the human body, for example: stimulation of nerves, muscles, and cardiac electrical activity. This phenomenon is used today in many medical applications. In this article, we discuss ways in which electromagnetic field affects the physiological and pathological processes in cells and tissues. This knowledge will help to better understand the electrophysiological phenomenon in connective tissue diseases and can bring new therapeutic strategies (in the form of "invisible drugs") for the treatment of rheumatic diseases?
\end{abstract}

Key words: electromagnetic field, reactive oxygen intermediates, redox.

\section{Introduction}

Although short-term exposure to an electromagnetic field (EMF) does not cause any apparent detrimental effects, exposure to higher levels of EMF causes acute and chronic effects in living organisms [1]. The mechanism of the EMF's influence on the body remains unclear. On the one hand, it is accepted that an EMF does not lead to genotoxic effects by direct transfer of energy to cells. But on the other, it seems that the effect of EMF exposure on living cells is indirectly mediated by increased halftime of reactive oxygen intermediates (ROI) [1, 2]. According to Varani et al. [3], from a clinical point of view, a clarification of the potential biological effect of electromagnetic exposure could facilitate the development of alternative treatments or the elaboration of novel promising therapeutic tools.

\section{Reactive oxygen intermediates and redox potential}

Numerous processes handle the generation of $\mathrm{ROI}$ in the body. The major source of ROI in the cells is mitochondria. These organelles accomplish cellular respiration and energy production (ATP) by the "electron transfer chain" mechanism. Since some electrons "leak" from the "electron transfer chain", those electrons interact with oxygen to produce ROI. It seems that $1-5 \%$ of the oxygen molecules may be converted to ROI [2]. Further sources of ROI are immune cells such as monocytes, macrophages and neutrophils. In phagocytic cells, ROI formation is part of the cascade of events in their antimicrobial action known as "the oxidative burst", which results from the assembly of a complex electron transport system in the cell mem-

\footnotetext{
*The first part of the paper is published in Reumatologia 2013; 51, 3: 195-201, and the second part in Reumatologia 2015; 53, 1: $40-45$.
}

Address for correspondence:

Michał Gajewski, Department of Biochemistry and Molecular Biology, Institute of Rheumatology, Warsaw, Poland,

e-mail: michal.gajewski@ir.ids.p

Submitted: 10.07.2015, Accepted: 28.08.2015 
brane. In these cells, the most important enzyme is the multimeric enzymatic complex of NADPH (nicotinamide adenine dinucleotide phosphate) oxidase complex and cytochrome $b$, which catalyses the univalent reduction of molecular oxygen to generate the ROI. The isoform of NADPH oxidase, localized in the membrane of non-phagocytic cells such as fibroblasts, endothelial cells and vascular smooth muscle cells, leads to much smaller quantities of ROI (one-third of that of neutrophils) and is mainly associated with cellular mechanisms involved in signal transduction cascades and regulatory processes $[1,2]$.

Since ROI can donate an electron to an appropriate acceptor (reduction reaction) or pair their unpaired electron by taking one from an appropriate donor (oxidation reaction), the living cells are in a stable state. This balance can be disturbed by increased ROI production, by decreased scavenging capacity of antioxidants or both. ROI have a significant influence on the redox state (reduction-oxidation) of the cells $[1,2,4]$.

\section{Redox potential in regulation of life cycle}

It is commonly accepted that the redox potential in the extracellular environment can dialog with the intracellular compartments of living cells. These observations suggested a novel role for the extracellular redox milieu in cell signaling and viability. The redox environment of the cells might determine whether cells will proliferate, differentiate, or die [5].

The reduction/oxidation state of living cells or tissues is a consequence of the continuous balance between the levels of oxidizing and reducing equivalents. The three most important redox-regulating systems within the cells are (NADPH/NADP+), thioredoxin (TRXred/TRXox) and glutathione (GSH/GSSG) $[2,5]$.

The most important system in regulating redox potential is GSH/GSSG, since the concentrations are about 500-1000 higher than TRX and NADPH. It was observed that under physiological conditions, the concentration of the reduced form of glutathione (GSH) was 10-100fold higher than the oxidized form (GSSG). Persistent production of $\mathrm{ROI}$ during oxidative stress and imbalance in the redox homeostasis leads to the formation of GSSG at the expense of GSH. This shift in the ratio of GSH/GSSG concentration changes the redox state to a more positive potential [4].

The changes in this ratio may govern several cellular reactions involved in signal transductions, cell cycle regulation and many other cellular processes. The changes in redox potently affect the cell proliferation rate and apoptosis. Upon the effects of oxidative stimuli, the GSH/GSSG ratio tends to decrease by raising the concentrations of glutathione disulfide or reducing the glutathione amount. For example, pro-oxidants increase the intracellular concentration of ROI, which can induce apoptosis. In contrast, antioxidants that serve as reducing agents have been shown to prevent apoptosis $[4,5]$.

Cell activation and proliferation are strongly dependent not only on levels of redox potential inside the cells, but also on the redox potential of the extracellular microenvironment. It was shown that the T cell differentiation into Th1, Th2, Th17 and/or Treg cells crucially depends on the activation of redox-sensitive signaling cascades. The oxidative conditions support Th1 cell development (proinflammatory), while reductive stress leads a shift to the Th2 type immune response. Finally, when the human organism is more oxidized, there is a change in the balance of Th cells in the immune system, which favors the Th1 arm of the immune system over the Th2 system [6].

However, as was shown by Jones et al. [7], human plasma GSH/GSSG levels within the body are controlled at a relatively constant redox state of $-137 \mathrm{mV}$. This balance is also related to aging. After age 50 , an oxidative shift of about $7 \mathrm{mV} /$ decade occurs over the next two decades, followed by a further decline to $-110 \mathrm{mV}$ in 70 to 85-year-olds [7]. Moreover, the changes of the half-cell reduction potential (Ehc) of GSSG/2GSH correlate with the biological status of the cell:

- proliferation is shown with a reduction potential of the glutathione half-cell in the range of Ehc -260 to $-221 \mathrm{mV}$,

- the differentiation is associated with a more positive potential from -221 to $-180 \mathrm{mV}$,

- apoptosis is triggered if Ehc rises in living cells from -180 to $-160 \mathrm{mV}$,

- necrosis occurs if Ehc rises above approximately $-160 \mathrm{mV}$,

- around -160 to $-150 \mathrm{mV}$ the redox buffering capacity of the GSH/GSSG system is permanently lost, as was summarized by Schafer and Buettner [4].

\section{Effects of electromagnetic field on the living cell}

From the biological point of view, the influence of EMF exposure depends on cell type and their homeostatic state. Moreover, it appears that EMF enhances the physiological functions of certain cell types including cell activation and proliferation. It is important to note that there are studies performed without observing any changes in the generation of ROI using other than immune relevant cells [1]. 
It is not known why various immune cell types respond to EMF with different efficacy, but it seems to be clear that several cell types are responsive to EMF by diverse mechanisms [1]. In a general view, the various cell types react differently to the same stimulus, and these cell type effects are redox status dependent $[1,2]$.

The responsiveness of the cells towards EMF may be correlated with the cell shape. For example, fibroblast cells ("stationary cells") are spindle-shaped, whereas blood cells ("inflammatory cells") are round in shape, and as was shown the "inflammatory cells" are more sensitive to EMF than "stationary cells" [1].

It was demonstrated that magnetic fields increase the halftime of ROI, and in consequence, the increased $\mathrm{ROI}$ concentration and dispersion increase the probability of cellular damage [1, 2]. In general, reactions in which $\mathrm{ROI}$ are involved become more frequent, increasing the possibility of cell and tissue damage. Some cellular components, processes, and systems may be affected by EMF exposure. Exposure to EMF alters certain cellular processes such as ROI production or extra- and intra-cellular emission. The influence of EMF may indirectly result in changes in DNA structure (strand breaks, chromosomal aberrations) and DNA repair processes leading to cytotoxic effects inducing cell death $[1,2,8]$.

Activation of immune cells (such as neutrophils) by short-term exposure to an EMF leads to phagocytosis and ROI emission. This pathway may be useful for specific therapeutic applications. An increase in the lifetime of ROI by EMF leads to persistently elevated ROI concentrations. The long-term EMF exposure leads to a chronically increased level of ROI, subsequently causing inhibition of the effects of the antioxidants. These processes results in an imbalance in redox state and enhance oxidative stress [1].

Lack of phagocytic activity and increased superoxide production after exposure to an EMF was detected in mouse macrophages and human monocytes [1]. These investigations demonstrated induction of $\mathrm{ROI}$ production, without a parallel increase of phagocytic machinery, indicating a specific activation of the NADPH oxidase after EMF exposure [1, 2]. Similar observations of activated rat peritoneal neutrophils were made by Roy et al. [9]. Exposure to an EMF resulted in an increased oxidative burst; however, the authors proposed an increased lifetime of $\mathrm{ROI}$ due to the EMF exposure as a possible mechanism [9].

Contrary to neutrophils or macrophages, lymphocytes do not respond to an EMF. Several studies have confirmed that an EMF does not induce DNA damage effects in human lymphocytes. This observation may be explained by the high homeostatic activity of these cells [10].

\section{The windows effect - fluctuations in electromagnetic field susceptibility}

It was found that the cytosolic concentration of NADPH fluctuated in a periodic manner in neutrophils and macrophages [11]. It was demonstrated that magnetic pulses may, on the one hand, increase the amplitude of the NADPH oscillations and production of ROI or, on the other hand, reduce the metabolic oscillations and diminish production of ROI [11, 12]. It was observed that NADPH oscillates in an approximate sinusoidal pattern with a period of 3-4 min. It was found that the production of ROI and NO was directly linked to NADPH oscillations [11].

Poniedziałek et al. [12] reported that the incubation of the unstimulated neutrophils in EMF decreases ROI production. The moderate decline of ROI level in unstimulated neutrophils may be attributed to the fall in biological activity of some enzymes catalyzing reactions connected with $\mathrm{ROI}$ production. Opposite results were obtained in stimulated neutrophils. Production of $\mathrm{H}_{2} \mathrm{O}_{2}$ in stimulated neutrophils increased after incubation in a low frequency EMF (compared to stimulated neutrophils not exposed to the field), whereas no such effect was observed after exposure of unstimulated neutrophils to low frequency EMF. These results suggested that a low-frequency EMF does not act as a direct stimulator of respiratory burst in neutrophils. This observation may be correlated with increased activity of one of the enzymes catalyzing the reaction cascade during the respiratory burst, the most likely candidate being NADPH oxidase [12].

Studies on the effect of pulsed magnetic fields on nitric oxide synthase (NOS) activity performed by Noda et al. [13] showed that the effect of EMF field depends on the magnetic induction. A substantial increase in NOS activity was observed for $0.1 \mathrm{mT}$ induction, whereas an induction value of 0.3 or $0.6 \mathrm{mT}$ showed no significant effect.

Experiments performed by Khadir et al. [14] demonstrated that the frequency of EMF may also differentially influence cell functions. The exposure of unstimulated neutrophils to a $60 \mathrm{~Hz}$ magnetic field did not affect the examined functions. In contrast, exposure of stimulated neutrophils to the magnetic field induced significantly increased ROI production. The mechanism by which EMF modifies cell functions is not yet precisely determined [14].

The fact that the effect of EMF on ROI production was observed only with some parameters of applied field indicates the existence of a "window effect", which was first described by Noda et al. [13]. Because magnetic fields easily penetrate tissue, these experiments sug- 
gested that ultra-low frequency pulsed magnetic fields may be useful to control cellular and tissue metabolism in vivo, and finally could have potential therapeutic value $[11,12,14]$.

\section{Possible role of electromagnetic field in regulating immune cell life cycle}

Several reports have suggested that the cell membrane is one of the most likely target sites of EMF-cell interaction. Calcium transport is a strong candidate for mediating the effect of EMF, due to its essential role in signal transduction and enzyme regulation [15-17].

Stimulation of neutrophils may induce a membrane potential change and translocation of proteins to the cell membrane. These events could be affected by EMF exposure. As was suggested by Walleczek [15], Ca²+ plays a significant role in signal transduction and the control of immune cell functions such as ROI production and emission. It was concluded that modulation of intracellular $\mathrm{Ca}^{2+}$ concentrations is possible only in stimulated immune cells [15].

It seems that voltage-gated calcium channels (VGCCs) play an essential role in cell response to an extremely low frequency EMF $(50 / 60 \mathrm{~Hz})$. Pall et al. [16] reported that VGCCs handle EMF effects. It was shown that the L-type or other VGCC blockers block or greatly lower diverse EMF effects. Voltage-gated properties of these channels may provide a biophysically plausible mechanism for the EMF biological effects. Moreover, the downstream responses to EMF exposures may be mediated through $\mathrm{Ca}^{2+} /$ calmodulin (CaM) stimulation of nitric oxide (NO) synthesis. This interpretation is supported by two well-documented examples of EMF effects. Firstly, the electromagnetic field stimulation of bone growth, modulated by the stimulation of osteoblasts, appears to involve an elevation the NO/protein kinase G pathway. Secondly, recent modeling has suggested that electrical effects may be very substantial and therefore may act to stimulate VGCCs directly [16].

Potentially therapeutic effects may be mediated through the $\mathrm{Ca}^{2+} / \mathrm{NO} / \mathrm{cGMP}$ (cyclic guanosine monophosphate)/protein kinase $G$ pathway, as was suggested by Pilla et al. [17] They proposed that an EMF can act as a first messenger in the CaM-dependent signaling pathways that orchestrate the release of cytokines in cellular responses to physiological stimuli. It seems that the EMF signals modulated CaM-dependent enzyme kinetics, produced several-fold increases in principal second messengers including NO and cGMP in chondrocyte and endothelial cell culture and, contrary to that, cyclic adenosine monophosphate (AMP) in neuronal cultures.
CaM antagonists blocked these effects, providing visible support for this mechanism [17].

It should be noted that this pathway is usually activated by increases in cytosolic concentrations of free $\mathrm{Ca}^{2+}$ in response to inflammation or oxidative stress. It appears that the CaM/NO/cGMP signaling pathway is a rapid response cascade that can significantly modulate cellular metabolism both in normal physiological conditions and in inflammation [18].

Contrary to that, Selvan et al. [19] demonstrated that pulsating EMF (PEMF) has a well-documented anti-inflammatory effect. They observed an elevated level of lipid peroxidation (LPO) and depletion of antioxidant enzymes in an adjuvant-induced arthritis model in rats. Also, plasma membrane $\mathrm{Ca}^{2+}$ ATPase (PMCA) activity was inhibited, while intracellular $\mathrm{Ca}^{2+}$ level, as well as prostaglandin E2 (PGE2) levels, were noted to be significantly elevated in blood lymphocytes of arthritic rats. Exposure of arthritic rats to PEMF at $5 \mathrm{~Hz} \times 4 \mu \mathrm{T} \times 90 \mathrm{~min}$ significantly reduces exudation. It was shown that this effect was a result of stabilization of cell membrane by PEMF, which restores activity of PMCA that handles the regulation of intracellular $\mathrm{Ca}^{2+}$ concentration. Restoration of PMCA keeps intracellular $\mathrm{Ca}^{2+}$ to an extremely low level, which results in inhibition of PGE2 biosynthesis [19].

In studies on antioxidant enzymes in mice, PEMF did not cause any changes [20]. It seems that the biological systems in a transition state were more sensitive to PEMF exposure than stationary regimes. It seems that arthritic animals are more sensitive to PEMF exposure. It is important to note that low-frequency and low-intensity PEMF is indeed beneficial in reducing inflammation without potential side effects [19].

Moreover, experiments performed by Juszczak et al. [21] showed that low-frequency PEMF $(50 \mathrm{~Hz}, 45+/-m T$, three times for 4 hours) stimulates induction of apoptosis and diminished necrosis of rat urothelial cells in culture. It seems that with EMF and/or PEMF it is possible to regulate redox balance of inflammatory cells and in this way control the physiological/pathophysiology state of other cells. Taken together, these observations suggested that EMF and/or PEMF could be developed as a real therapy for rheumatoid arthritis [19].

\section{Conclusions}

Reasons why the acute inflammatory phase does not convert into the reconstruction process, but becomes chronic inflammation instead, are complex. One of the factors responsible for that is impaired energy flow to the immune system and disturbed parasympathetic nervous balance [23]. This results in disturbance in redox homeostasis and depletion of available energy 


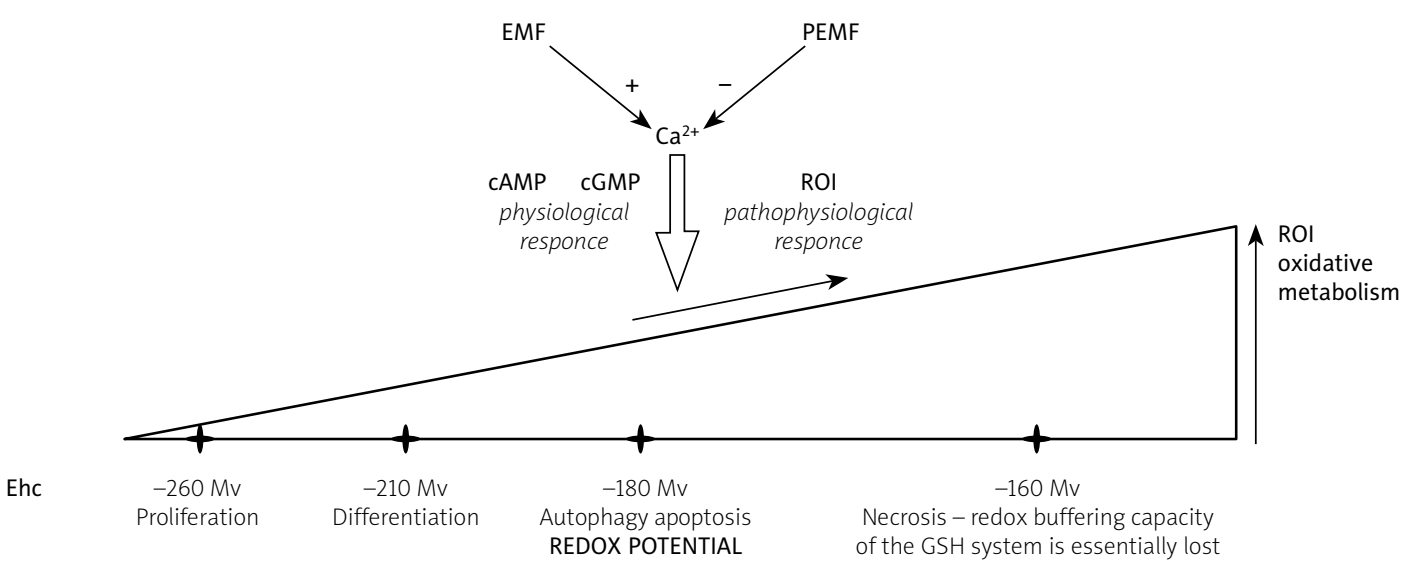

Ehc - half cell reduction potential; $m V$ - microvolt; $C A M P$ - cyclic adenosine monophosphate; cGMP-cyclic guanosine monophosphate; $N O$-nitric oxide; $R O I$ - reactive oxygen intermediates; EMF-electromagnetic field; PEMF-pulsating electromagnetic field

Fig. 1. Possible mechanism of the magnetic field influence on the course of the life cycle of cells by the impact of their redox potential.

stores [24]. May the better understanding of electrophysiological processes of diseased tissue bring new therapeutic strategies?

It is commonly accepted that changes in the redox state determine whether cells die as well as the kind of death: necrosis or apoptosis. Ultimate "survival" or "death" signals depend on the redox signaling, transcription regulation of several redox-sensing genes and DNA repair processes [25].

Apoptosis occurs with low or moderate oxidative stress and redox potential changes, whereas necrosis results from severe oxidative challenges that overcome the cellular antioxidant defenses and energy-transducing pathways [26]. Common inducers of apoptosis include $\mathrm{ROI}$ and $\mathrm{Ca}^{2+}$. These factors give rise to signals to cells resulting in apoptotic or necrotic cell death [25].

It may be assumed that cells, during the cell life, transform from the proliferation and differentiation stage to autophagy (auto-phagocytosis intracellular components), apoptosis (controlled death, without inflammatory events), and finally to necrosis (uncontrolled death with inflammatory events). Extracellular signals induced by EMF or PEMF may influence (Fig. 1) such genetic-dependent phenomena as autophagy ("survival signals"), apoptosis ("safety death signals") or necrosis ("death signals").

We can hypothesize that if it was possible to regulate the production of $\mathrm{ROI}$ and $\mathrm{Ca}^{2+}$ metabolism by EMF and/or PEMF in inflammatory cells, it might be possible to inhibit the "conversion" and "transition" of the signals from the signal "survival" to the signal "death" by the redox regulating system. It could be suggested that such changes in redox potential, mediated by the magnetic field, may be partially detox of surrounding tissues and cells. It can be concluded that EMF and PEMF influ- ence of genetically-dependent phenomena fits into the problems of molecular oncology.

\section{Acknowledgments}

We gratefully thank dr Agnieszka Paradowska-Gorycka for language correction of the manuscript.

The authors declare no conflict of interest.

Article prepared as a part of statutory activities of the Institute of Rheumatology no. S/2015.

\section{References}

1. Simko M, Mattsson MO. Extremely low frequency electromagnetic fields as effectors of cellular responses in vitro: possible immune cells activation. J Cell Biochem 2004; 93: 83-92.

2. Simko M. Cell specific redox status is responsible for diverse electromagnetic field effects. Curr Med Chem 2007; 14: 1141-1152.

3. Varani K, Gessia S, Merighia S, et al. Alteration of A3 adenosine receptors in human neutrophils and low frequency electromagnetic fields. Biochem Pharmacol 2003; 66: 1897 1906.

4. Schafer FQ, Buettner GR. Redox environment of the cell as viewed through the redox state of the glutathione disulphide/ glutathione couple. Free Rad Biol Med 2001; 30: 1191-1212.

5. Filomeni G, Rotilio G, Ciriolo MR. Cell signaling and the glutathione redox system. Biochem Pharmacol 2002; 64: 1057-1064.

6. Gostner JM, Becker K, Fuchs D, et al. Redox regulation of the immune response. Redox Rep 2013; 18: 88-94.

7. Jones DP, Mody Jr VC, Carlson JL, et al. Redox analysis of human plasma allows separation of pro-oxidant events of aging from decline in antioxidant defences. Free Rad Biol Med 2002; 33: $1290-1300$ 
8. Simko M. Induction of cell activation processes by low frequency electromagnetic fields. Scientific World Journal 2004; 4 (S2): 4-22.

9. Roy S, Noda Y, Eckert V, et al. The phorbol 12-myristate 13-acetate (PMA)-induced oxidative burst in rat peritoneal neutrophils is increased by a $0.1 \mathrm{mT}(60 \mathrm{~Hz})$ magnetic filed. FEBS Lett 1995; 376: 164-166.

10. Scarfi MR, Lioi MB, Zeni O, et al. Lack of chromosomal aberration and micronucleus induction in human lymphocytes exposed to pulsed magnetic fields. Mutat Res 1994; 306: 129-133.

11. Rosenspire AJ, Kindzelskii AL, Simon BJ, et al. Real-time control of neutrophil metabolism by very weak ultra-low frequency pulsed magnetic fields. Biophys J 2005; 88: 3334-3347.

12. Poniedzialek B, Rzymski P, Nawrocka-Bogusz H, et al. The effect of electromagnetic field on reactive oxygen species production in human neutrophils in vitro. Electromagn Biol Med 2013; 32: 333-341.

13. Noda Y, Mori A, Liburdy RP, et al. Magnetic field and lipoic acid influence the respiratory burst in activated rat peritoneal neutrophils. Pathophysiology 2000; 7: 137-141.

14. Khadir R, Morgan JL, Murray JJ. Effects of $60 \mathrm{~Hz}$ magnetic field exposure on polymorphonuclear leukocyte activation. Bioch Biophys Acta 1999; 1472: 359-367.

15. Walleczek J. Electromagnetic field effects on cells of the immune system: the role of calcium signaling. FASEB J 1992; 6 : 3177-3185.

16. Pall ML. Electromagnetic fields act via activation of voltage-gated calcium channels to produce beneficial or adverse effects. J Cell Mol Med 2013; 17: 958-965.

17. Pilla AA, Fitzsimmons R, Muehsam D, et al. Electromagnetic fields as first messenger in biological signaling: application to calmodulin-dependent signaling in tissue repair. Biochim Biophys Acta 2011; 1810: 1236-1245.

18. Pilla AA. Mechanisms and therapeutic application of time varying and static magnetic fields. In: Biological and Medical Aspects of Electromagnetic Field, Barnes F, Greenbaum B (eds.). CRC Press, Boca Raton FL 2006: 351-411.

19. Selvam R, Ganesan K, Narayana R, et al. Low frequency and low pulsed electromagnetic field exerts antiinflammatory effect through restoration of plasma membrane calcium ATPase activity. Life Sci 2007; 80: 2403-2410.

20. Eraslan G, Bilgili A, Akdogan M, et al. Studies on antioxidant enzymes in mice exposed to pulsed electromagnetic fields. Ecotoxicology Environm Safety 2007; 66: 287-289.

21. Juszczak K, Kaszuba-Zwoinska J, Thor PJ. Pulsating electromagnetic field stimulation of urothelial cells induces apoptosis and diminished necrosis; new insight to magnetic therapy in urology. J Physiol Pharmacol 2012; 63: 397-401.

22. Kubat NJ, Moffett J, Fray LM. Effect of pulsed electromagnetic field treatment on programmed resolution of inflammation pathway markers in human cells in culture. J Inflammation Res 2015; 8: 59-69.

23. Gajewski M, Rzodkiewicz P, Wojtecka-Lukasik E. The role of physiological elements in the future therapies of rheumatoid arthritis. II. The relevance of energy redistribution in the process of chronic inflammation. Reumatologia 2015; 53: 40-45.

24. Gajewski M, Rzodkiewicz P, Maslinski S, Wojtecka-Lukasik E. The role of physiologic issues in future therapy of rheumatoid arthritis. I. Chronic inflammation toward action of parasympathetic system. Reumatologia 2013; 51: 195-201.

25. Das DK, Maulik N. Preconditioning potentiates redox signaling and converts death signal into survival signal. Arch Biochem Biophys 2003; 420: 305-311.

26. Leist $M$, Single B, Castoldi AF, et al. Intracellular adenosine triphosphate (ATP) concentration: a switch in the decision between apoptosis and necrosis. J Exp Med 1997; 185: 1481-1486. 